\title{
How Important are Risk Factors in Alzheimer's Disease?
}

\author{
Ming Chen ${ }^{\mathrm{a}, \mathrm{b}, *}$ and Hugo L. Fernandez $\mathrm{z}^{\mathrm{a}, \mathrm{c}}$ \\ ${ }^{a}$ Neuroscience Research Laboratory, Medi- \\ cal Research Service (151), Bay Pines VA, \\ Medical Center, Bay Pines, FL, USA \\ ${ }^{b}$ Department of Pharmacology and Thera- \\ peutics, University of South Florida College \\ of Medicine, Tampa, Florida USA \\ ${ }^{c}$ Department of Neurology and Department \\ of Physiology and Biophysics, University of \\ South Florida College of Medicine, Tampa, \\ FL, USA
}

Alzheimer's disease (AD) has traditionally been considered as a natural event in aging by the general public. But this simple perception is apparently problematic because it cannot explain why only some people, but not others, develop $\mathrm{AD}$ at the same age. Indeed, the commentaries by Drs. Mattson (1), Price et al. (2) and Khachaturian (3) all argue against this view, yet they recognize that aging and $\mathrm{AD}$ are strongly associated.

Perhaps because of the problems of the traditional view, much of the modern $\mathrm{AD}$ research has based on the concept that aging and $\mathrm{AD}$ are "two independent processes". But, after perhaps the most intensive studies for decades, there has been little if any evidence indicating a direct participation of a conventional pathogen(s) in the development of most cases of late-onset sporadic AD (which does not include early-onset and some other types of dementia). At the same time, how-

\footnotetext{
* Corresponding author: Ming Chen, Ph.D., Neuroscience Research Laboratory, Medical Research Service (151), Bay Pines VA Medical Center, Bay Pines, Florida 33744, USA, Fax: +1 727398 9467, E-mail: michen@com1.med.usf.edu
}

ever, it has been established that several "risk factors" play a critical and common role in AD, a situation similar to many other age-related conditions.

Under this circumstance, we think that it is necessary to reconsider the roles played by the risk factors. Accordingly, we proposed a third model, that is, risk factors under the condition of advanced aging can play a primary role in lateonset sporadic AD. Specifically, this model first distinguishes "neurodegeneration" from "AD", two widely interchangeably-used terms: one is a necessary event in aging, the other is the condition at its extreme or excessive stages. The model then separates late-onset sporadic $\mathrm{AD}$ from other dementia cases (mostly early-onset). Under this definition, we consider aging (neurodegeneration) as a necessary (but not sufficient) condition which provides the stage for AD development, whereas other risk factors largely determine the onset age (relative to vital organ failures) and the severity of the cognitive decline (whether it will reach the clinical definition).

Since this model is a departure from the current views about the disease, it would naturally surprise some investigators. Indeed, it was a surprise to ourselves as well when it first came as an inevitable outcome of our analytic reasoning. Now, this model, like any other hypotheses, is subject to criticisms and testing, which will eventually allow a more correct model to emerge for the disease. Despite that the evidence available to support our model is considered by the commentaries as not compelling (1-3), one still cannot dismiss it as "incorrect" at the present time, because such a conclusion can be logically drawn only after a causal and common pathogen 
has been found. Obviously, additional studies will be required before the complex relationship between aging and AD is fully understood.

The fact that some people remain healthy beyond the tenth decade does not argue against our view that late-onset sporadic $\mathrm{AD}$ is largely a natural event intensified by risk factors and it approaches necessity at very old age. This argument parallels that for human death, a necessity, and while some die at ages 80-90 from natural events, others remain healthy at the same or more advanced age.

If vital organ failure is a necessity, then why is the same concept for the brain so difficult to accept $(2,3)$ ? Probably because it seemingly confronts with our belief that "AD is preventable". These two concepts, however, are not contradictory. "AD is preventable" means that it can be postponed to not occur before the vital organ failure, a practical goal since the vital organ lifespan is limited. But the concept of "necessity" is a logical extension under the condition "if one lives long enough" (unlimited). In other words, AD is a "necessity" in concept but also "preventable" in real life. However, we emphasize the "necessity" concept because it is the logic basis to understand the nature of $\mathrm{AD}$ as fundamentally different from that of AIDS or cancer.
$\mathrm{AD}$ is a devastating disease, but not all diseases are caused by conventional pathogens. While pathogens are important in the age-related dementias as a whole, our model suggests to search and target factors other than conventional pathogens in $\mathrm{AD}$. $\mathrm{AD}$ is unique in affecting the most exquisite part of body functions, so conceivably, its contributing factors may turn out to be equally unique and exquisite. How exquisite can they be? Perhaps as exquisite as " $\mathrm{Ca}^{2+}$ pulse frequency" changes (4). How unique? Perhaps as unique as, for example, "story-telling with grandchildren". This works by activating the oldest memories (the most important memories) by selectively exciting the oldest synapses thus differing from other social activities, but is quietly disappearing today. If we consider the oldest-old cells as severely overburdened camels, then some "straws" might give rise to an unexpected effect.

Recognizing the impact of such factors and effective intervention require the participation of society as a whole. Calling AD "independent of aging" may be necessary to attract the attention of the society at large, but may not accurately guide the scientific investigation at its starting point. Indeed, the NIH goal for $\mathrm{AD}$ research aims to postpone its onset age by $5-10$ years in the near future is similar to our argument. Nota-

Table 1

Which questions are more likely to lead to correct answers in AD research?

\begin{tabular}{|c|c|}
\hline Most Frequently Asked Questions & Alternative ways to ask the same questions \\
\hline What is wrong in $\mathrm{AD}$ ? & What makes AD unique to other diseases? \\
\hline $\begin{array}{l}\text { Why do plaques and tangles accumulate } \\
\text { in the brain? }\end{array}$ & $\begin{array}{l}\text { Why do they accumulate together with many other aging markers in } \\
\text { the body? }\end{array}$ \\
\hline What causes plaques and tangles? & $\begin{array}{l}\text { What is the common cause for all aging markers? Why do they } \\
\text { occur in essentially everyone and at about the same time? }\end{array}$ \\
\hline $\begin{array}{l}\text { How many processes have gone wrong } \\
\text { in } \mathrm{AD} \text { brain? }\end{array}$ & $\begin{array}{l}\text { What is the central factor controlling neurotransmission and mem- } \\
\text { ory? What happened to that factor during aging? }\end{array}$ \\
\hline Why do cells die? & $\begin{array}{l}\text { Why do the oldest-old cells die in the apparent absence of dramatic } \\
\text { insults in sporadic AD? }\end{array}$ \\
\hline What can break the back of a camel? & $\begin{array}{l}\text { Is AD camel the same with other camels? } \\
\text { What should we look for, "huge stones" or "straws"? }\end{array}$ \\
\hline
\end{tabular}


bly, the logic basis for this practical goal is the perception of $\mathrm{AD}$ as an essentially natural event (otherwise the goal would be to cure or eliminate it like AIDS).

How to postpone AD? Targeting oxidative stress, apoptosis, $\mathrm{Ca}^{2+}$ rises, or glucose transport problem (1) is not the answer, because a more important question here is where these damages come from. Yes, they can come from gene mutations, but what about the sporadic cases who do not have gene mutations? To this critical and difficult question, we proposed that in those cases they, most likely, come from risk factors.

It is widely believed that plaques and tangles may cause these cellular damages. But we contended that plaques and tangles are the natural products of aging and are originated from metabolic inefficiency (completely different from HIV or cancer). Now, are they the natural products, or not?

Yet, is targeting the cellular damages the only option before us? The current knowledge that physical/brain exercises and replenishing hormones, growth factors and vitamins are effective clearly suggests that re-activating the normal life-supporting metabolisms is an important research direction to postpone $\mathrm{AD}$, like many other age-related diseases.

Dr. Mattson stated that our view that $\mathrm{Ca}^{2+}$ signaling potency is reduced in aging is "directly against an overwhelming body of evidence" (1). We however found that this "overwhelming body of evidence" is directly against a common observation in the first place: $\mathrm{Ca}^{2+}$-mediated processes in the body all reduce their potency in aging (cell growth, muscle contraction, neurotransmission, etc.). We think that this is because these studies have not taken into account of a key fact that $\mathrm{Ca}^{2+}$ exerts its effects by changing its pulse frequency and amplitude, not the usually measured "concentrations". Indeed, by considering this intriguing mechanism, the two opposing views in this central matter can be partly conciliated (4). The issue also pertains to some basic concepts in $\mathrm{Ca}^{2+}$ signaling, so we will further explore it (5).

When these issues were reconsidered, it came to our attention that some controversies can be traced to the ways in which the questions were initially asked. Table 1 lists some of these questions. We believe that asking right questions is perhaps the most important starting point to reach correct answers.

\section{REFERENCES}

1. Mattson MP, Risk factors and mechanisms of alzheimer's disease pathogenesis: obviously and obviously not, J Alzheimer Dis 2(2) 109-112.

2. Price JL, Phil D, Rubin, EH, Morris JC, Commentary: Revisiting Alzheimer's disease from a new prospective: can "risk factors" play a key role, J Alzheimer Dis 2(2) 113-114.

3. Khachaturian ZS, Aging: a cause or a risk for AD? J Alzheimer Dis 2(2) 115-116.

4. Chen $\mathrm{M}$, Fernandez $\mathrm{HL}, \mathrm{Ca}^{2+}$ signaling downregulation in ageing and Alzheimer's disease: why is $\mathrm{Ca} 2+$ so difficult to measure?, Cell Calcium 26 (1999) 149-154.

5. Chen M, Fernandez HL, Postpone Alzheimer's disease by targeting $\mathrm{Ca}^{2+}$ "pulse frequency": important theoretic issues, (submitted). 